\title{
Medición y evaluación en comunicación estratégica. Caso de estudio Ministerio del Ambiente del Ecuador
}

\author{
Measurement and evaluation in strategic communication. Case study \\ Ministry of Environment of Ecuador
}

\author{
Karen Cesibel VALDIVIEZO ABAD \\ Universidad Técnica Particular de Loja, Ecuador \\ kcvaldiviezo@utpl.edu.ec \\ María Teresa SOSA \\ Universidad Técnica Particular de Loja, Ecuador \\ teresasosa2008@hotmail.com
}

Recibido: 14 de agosto de 2015

Aceptado y Publicado: 15 de septiembre de 2015

\section{Resumen}

En la actualidad y dado el alto nivel de complejidad y la gran velocidad a la que las distintas organizaciones han evolucionado es esencial el reconocimiento de la comunicación como herramienta práctica y eje de competitividad de las mismas. Un elemento que ha contribuido ciertamente a la construcción de las relaciones humanas debe ser evaluado y medido con el objetivo de conocer la verdadera aportación de esta a las actividades desempeñadas por la organización de forma general y en todos sus niveles.

El presente trabajo se enfoca en el análisis de la planificación de comunicación estratégica del Ministerio del Ambiente (MAE), a través de la gestión de la Dirección de Comunicación en relación a la campaña "Tradición y Conservación van de la mano", la misma que será evaluada a través de la aplicación del modelo denominado "Communication Managment Bridge".

Con este sistema de medición se pretende conocer si los objetivos de comunicación de la organización seleccionada están vinculados directamente a los objetivos generales de la campaña. Considerando que los objetivos pueden ser considerados medios necesarios para la obtención de resultados deseados, los mismos que generarán grandes aportaciones dependiendo de su claridad y calidad informativa. 


\section{Abstract}

At present and given the high level of complexity and the great speed at which different organizations have evolved is essential recognition of communication as a practice and competitiveness axis of the same tool. One element that has certainly contributed to the construction of human relations should be assessed and measured in order to know the real contribution of this to the activities performed by the organization in general and at all levels. This paper focuses on the analysis of strategic communication planning, Ministry of Environment (MAE), through managing the Communication in connection with the campaign "Tradition and conservation go hand in hand", the same as It will be evaluated through the application of the model called "Communication Managment Bridge".

With this measurement system is intended to determine whether the communication objectives of the selected organization are directly linked to the overall objectives of the campaign. Whereas the objectives may be considered necessary means to obtain desired results, they will generate great contributions depending on the clarity and quality of information.

Palabras Clave: Comunicación estratégica, Medición, Evaluación, Objetivos, Plan Estratégico.

Key Words: Strategic Communication, Measurement, Evaluation, Objectives, Strategic Plan.

\section{Introducción}

La presente investigación se enfoca en el análisis de la planificación estratégica de comunicación en el Ministerio del Ambiente del Ecuador, a través de la gestión de comunicación realizada desde la Dirección de Comunicación, en relación a la campaña "Tradición y Conservación van de la mano", que se desarrolló en el 2013 y que hasta la actualidad permanece vigente y que se desarrolla cada año, en el marco de la celebración religiosa del Domingo de Ramos, enfocada a disminuir especies de la palma de cera, árboles de más de cien años que son deforestados, afectando el ecosistema. Esta campaña tiene como principal objetivo sensibilizar a la comunidad católica y a la sociedad en general, sobre la importancia de conservar la palma de cera y evitar la extinción de dos especies de aves que habitan y anidan en ella, que son: el loro orejiamarillo y el perico cachetidorado. 
Los medios y técnicas que utiliza la institución, coordinada por la Dirección de Comunicación, es a través de canales de comunicación como vocería en medios, redes sociales, presencia en ferias, free press, acciones de Relaciones Públicas y la colaboración directa de otras instituciones como la Iglesia Católica y la Policía Nacional, que ayudan con la educación ambiental y los controles respectivos.

Al ser una campaña de bajo presupuesto, se financia a través de acciones de autogestión; sin embargo el impacto que se genera es alto al ser aplicada a nivel nacional, logrando así un éxito de la misma. Cada año, esta campaña se planifica con dos meses de anterioridad a la celebración del Domingo de Ramos.

Se procedió a evaluar con la aplicación del modelo denominado "Communication Management Bridge", que consta de tres fases: estratégica, táctica y operativa.

En la actualidad y considerando el alto nivel de complejidad y velocidad a la que las organizaciones van evolucionando, es esencial reconocer que la comunicación es una herramienta de mucha importancia contribuye a la construcción de las relaciones humanas. También es necesaria que la comunicación sea medida y evaluada, con el objetivo de conocer el aporte que generan las actividades ejecutadas y el cumplimiento de los objetivos planteados.

El objetivo principal de la presente investigación fue determinar la efectividad que tuvo la Planificación Estratégica en Comunicación, en el Ministerio del Ambiente, aplicando la función de medición y evaluación de resultados, que permitió determinar el aporte de la gestión de comunicación a los objetivos de la organización, el estado de cumplimiento y el valor agregado que generó. Los resultados finales evidenciaron el impacto que tuvo en la sociedad en general, sin que exista ninguna alteración en cuanto a la costumbre religiosa. 


\section{Objetivos}

En concreto se plantaron los siguientes objetivos:

\section{Objetivo General}

Demostrar a través del estudio de caso del Ministerio del Ambiente, Dirección de Comunicación, durante el período 2013 - 2014, que la Planificación Estratégica en Comunicación, incluida la función de medición y evaluación de resultados, permiten determinar el aporte de la gestión de comunicación a los objetivos de la organización.

\section{Objetivos Específicos}

- Realizar una aproximación a la realidad de la organización (Ministerio del Ambiente del Ecuador).

- Analizar en el caso de estudio, mediante la aplicación de un modelo de medición y evaluación al programa de comunicación más importante durante el 2013; la contribución de la comunicación a los objetivos de la organización.

- Aportar mejoras para la planificación estratégica en la organización, con especial énfasis en la función de evaluación.

\section{Marco Teórico: La comunicación en las organizaciones}

La comunicación es la esencia de la actividad organizativa y es fundamental para un buen funcionamiento organizacional. Una buena comunicación mejora la competitividad de la organización, facilita el logro de los objetivos y metas establecidas. Anthony Robbin, explica que para comunicarse de forma efectiva debemos darnos cuenta que cada uno de nosotros percibe el mundo de diferente manera y debemos utilizar el conocimiento como guía para comunicarnos con los demás (Comunicación Asertiva, 2014). 
Las distintas relaciones entre personas y los grupos que nos rodean se han generado a través de la comunicación, la misma que facilita la actividad humana; los medios de comunicación han evolucionado en concordancia al nivel de complejidad que han adquirido las organizaciones y las relaciones que surgen tanto a nivel interno como externo.

La consolidación de un sistema de comunicación eficiente dentro de una organización puede determinar la prevalencia de la misma y permitirá el logro de los objetivos planteados. La planificación estratégica en comunicación forma parte de un conjunto de estrategias que funcionan paralelas a un proyecto, y al ser elaboradas correctamente, se convierten en un sistema de comunicación que ayuda al desarrollo del proyecto y alcance los resultados establecidos.

Álvarez Nobell A. (Medición y Evaluación en Comunicación, p. 10), menciona que la función de la comunicación en las organizaciones consiste en planificar, gestionar y evaluar las relaciones con los públicos internos y externos, en relación directa a los objetivos de la organización y el entorno económico, político, cultural, social y medioambiental al que pertenece.

La planificación estratégica en comunicación forma parte de los procesos de dirección en las organizaciones, transformándose en un vector que aporta un valor fundamental en escenarios ceñidos por la digitalización tecnológica que modifican los procesos comunicativos en torno a un mundo virtual cada vez más sistemático (Bertalanfy, 1976).

En este mismo sentido, evaluar los objetivos es absolutamente vital si el programa que se está planificando pretende ser demostrable. En la planificación estratégica, el objetivo, es entendido como un primer planteamiento para hacer algo, que siempre será medible y alcanzable en un determinado tiempo. Ello será la clave del arco del plan de comunicación. 
El único propósito de la primera etapa - la investigación y el análisis previo de la situación no será otro que definir claramente un objetivo el cual, a su vez, dará origen al diseño de las etapas posteriores, el objetivo permitirá establecer una estrategia para el conjunto de acciones de comunicación, es decir, la meta a lograr.

Complementario a ello, todas las acciones de comunicación desarrolladas deben ser evaluadas y los resultados deben ser visibles para quienes están liderando la organización. Álvarez (2011), propone un modelo integral de gestión de la medición y evaluación en comunicación estratégica, el "Communication Management Bridge", donde la función de evaluación en la planificación estratégica de la comunicación debe ser la participativa con el resto de los niveles de la organización, es decir tanto los niveles operativos como los directivos.

Es importante, proponer una función de comunicación estratégica que sirva de enlace entre la misión y los propósitos de la organización, (llevada por directivos) y la gestión de comunicación (técnicas, acciones y herramientas). En este proceso, la persona encargada de la comunicación será responsable de la dinámica organizacional y de llevar a cabo los procesos de integración técnica, para lo cual tendrá que conocer, estar preparado, tener dominio de las lógicas de gestión adoptadas por la organización y su concepción de resultado.

El Modelo "Communication Management Bridge", plantea para la función de evaluación en la planificación estratégica de la comunicación, una matriz elaborada a partir de un conjunto de criterios organizados en tres fases integradas: estratégica, táctica y operativa.

La fase estratégica responde a la función de planificación de la evaluación que lleva adelante el responsable de comunicación en interacción con los demás departamentos y el 
management de la organización. Esta fase tiene lugar cuando la organización define las políticas, su planificación estratégica, los planes de acción y los presupuestos.

La fase táctica corresponde al diseño de la medición, nos permitirá hacer operativas las definiciones de la fase estratégica para poder instrumentar la medición.

En la fase operativa se miden las actividades y acciones en función de los objetivos establecidos y las variables elegidas a ser gestionadas y evaluadas.

\section{Metodología}

Los métodos de investigación utilizados para la presente investigación fueron de tipo exploratorio y descriptivo, mediante la aplicación del modelo denominado "Communication Management Bridge". Así mismo se aplicó un método cualitativo, que incluye la integración de técnicas de análisis documental, entrevista semi-estructurada y observación directa enfocadas al desarrollo de la planificación estratégica de la campaña caso de estudio.

En el análisis documental se hizo una recopilación de información utilizando medios electrónicos, documentos, folletos institucionales. Se aplicó una entrevista semi-estructurada a María Augusta Yépez, Coordinadora de Campañas, el proceso que se aplicó en esta entrevista fue a través de un esquema de preguntas abiertas preestablecidas.

\section{Resultados}

Los resultados se analizan en función de las tres fases que considera el modelo "Communication Management Bridge": estratégica, táctica y operativo.

En la fase estratégica, se hizo una determinación del modelo de gestión organizacional para ello se analizó el perfil de gestión organizacional, donde se observó y determinó que la institución caso de estudio es del sector público, de servicio, sin fines de lucro, encargado de la gestión ambiental de todas y todos los ecuatorianos. Para los procedimientos 
organizacionales de control de gestión existentes cuenta con una Dirección de Comunicación que se encarga de la coordinación de la comunicación y de la campaña caso de estudio que contó con un plan de comunicación.

Los medios y canales de comunicación que utilizó la campaña Tradición y Conservación van de la mano fueron: redes sociales, como principal medio de comunicación, la televisión, la radio, las ferias, capacitación en escuelas y colegios a nivel nacional. Además se logró la participación total de las Direcciones y Subsecretarías que conforman el Ministerio del Ambiente a nivel nacional, así como de otras organizaciones externas como la Curia. Lo que se buscó fue impactar en la opinión pública desde la difusión previa hasta la aparición en los medios de comunicación y generar ruido en redes sociales. Paralelo a ello, se brindaron charlas de sensibilización en diferentes escuelas y colegios con el objeto de concienciar a la ciudadanía.

En la fase táctica se conceptualizaron variables de interés a evaluar de los objetivos de resultado. Las variables a considerar fueron: imagen positiva, transparente, tanto a nivel interno como externo del Ministerio del Ambiente; identidad para conocer si el MAE asume el reto de rediseñar su institucionalidad y reafirmar su rol de Autoridad Ambiental, basado en su misión, visión, y valores; público, para conocer si la campaña llega a toda la ciudadanía ecuatoriano a través de los diferentes medios y canales de comunicación.

En la fase operativa se establecieron niveles y dispositivos de medición en función a cada objetivo propuesto.

Clipping: Se realizará un mes después de cada charla en cada provincia, luego se realizará un análisis de resultados cada año, por el mes de mayo y junio, que serían los meses en los cuales se podría evaluar si se logró cumplir con el objetivo propuesto. 
Encuesta a participantes: Luego de la charla realizada por los funcionarios del Ministerio del Ambiente, se procederá a una encuesta a los participantes, para conocer el grado de satisfacción respecto a la misma.

Redes Sociales: seguirá siendo el medio principal de información que el Ministerio del Ambiente utilice, y la ciudadanía también para informarse.

Focus group: permitirá conocer, analizar y evaluar los resultados que se ha logrado con la participación de todas las Direcciones y Subsecretarias que están a cargo de la campaña.

Observación participante: el MAE cuenta con una estructura orgánica-funcional, que permite que la Dirección de Comunicación elabore los planes estratégicos para cada campaña o proyecto, y se pueda garantizar su cumplimiento, promoviendo un ambiente sano y sustentable.

Registros reales de consumo: tiene que emprenderse una planificación estratégica y una coordinación general adecuada para definir y determinar la información e investigación válidas y pertinentes para mejorar la situación ambiental, y lograr la participación y concienciar a la ciudadana para conseguir estos cambios ambientales.

Datos arrojados por Diario El Comercio en el Ecuador, dan a conocer que los ramos de palma decomisados desde el 2011 hasta el 2014 ha disminuido. En al siguiente tabla se puede visualizar las cifras. 
Tabla 1. Disminución de ramos de palma

\begin{tabular}{|l|c|c|c|c|c|c|c|}
\hline DATOS & 2011 & $\mathbf{2 0 1 2}$ & Diferencia & $\mathbf{2 0 1 3}$ & Diferencia & $\mathbf{2 0 1 4}$ & Diferencia \\
\hline $\begin{array}{l}\text { Ramos de Palma } \\
\text { decomisados a escala nacional }\end{array}$ & 1400 & 820 & -580 & 500 & -320 & 280 & -220 \\
\hline
\end{tabular}

Fuente: El Comercio (2013)

Elaboración propia

Gráfica 1. Cifras de disminución de ramos de palma

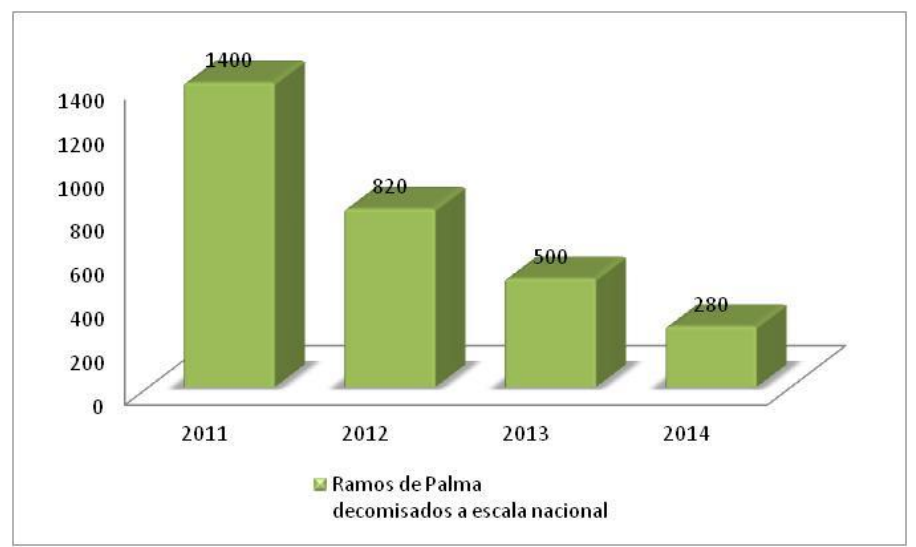

Fuente: El Comercio (2013)

Elaboración propia

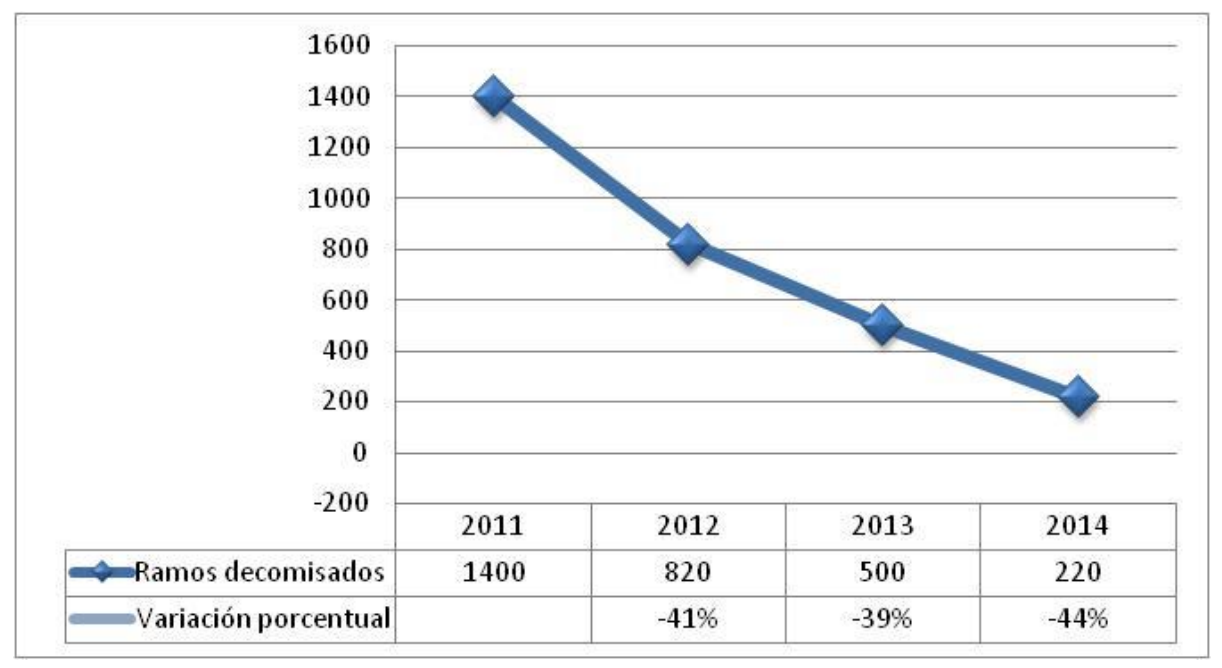

Fuente: El Comercio (2013)

Elaboración propia 
En referencia a la investigación efectuada se puede observar en los cuadros estadísticos antes señalados los resultados positivos obtenidos en la campaña "Tradición y conservación van de la mano", ya que se evidencia en la reducción considerable del $44 \%$ promedio de la tala de la palma de cera, que se lo puede evidenciar cada año y que a la vez se promueve el uso de materiales alternativos que no afectan el medio ambiente, que es el principal objetivo de esta campaña. Algo destacable de esta campaña es que no ha existido alteración en la costumbre religiosa, que se celebra previo a Semana Santa, sino que se ha provocado un cambio de palma de cera a materiales alternativos que no afecten la vida silvestre y ambiental.

\section{Discusiones y conclusiones}

Las instituciones públicas del Estado Ecuatoriano están en un proceso de cambio en cuanto a la imagen institucional y de servicio a la ciudadanía para el proceso del concepto del "Buen Vivir", se evidencia un antes y un después de las organizaciones públicas. En el caso del Ministerio del Ambiente del Ecuador, se pudo evidenciar los resultados positivos que se logró en esta campaña pero todavía existen falencias en cuanto a la planificación estratégica en comunicación, que a futuro de deben mejorar para el logro de los procesos de planificación de la comunicación de la organización.

Es necesario que exista entre la Dirección de Comunicación del MAE una mayor sinergia con las áreas involucradas en la campaña. Es primordial incorporar en este proceso un registro de control y seguimiento de la información, en cuanto a documentación que permita mayor desarrollo de la campaña, para mejorar el cumplimiento integral de los objetivos propuestos.

El desarrollo de buenas prácticas de comunicación, capacitación e información permitirán cumplir de forma adecuada el plan estratégico de comunicación, esto significaría un mejoramiento para la Dirección de Comunicación, y por ende a la organización, a través de 
la ayuda de una asesoría especializada que les permita implementar programas y directrices orientadas a los lineamientos de la institución.

En el caso de la investigación realizada en el Ministerio del Ambiente, existe un plan estratégico de comunicación, sin embargo el desarrollo y cumplimiento del mismo no se lo practica en su totalidad. Se enfatiza la idea que el proceso de comunicación en una organización representa la herramienta más eficaz para alcanzar las metas establecidas, ya que empleada correctamente la comunicación estimula la participación y favorece la unificación de criterios con miras a un bien común. Las organizaciones hoy en día buscan comunicarse de manera eficaz para establecer propósitos y aplicar correctivos.

\section{Bibliografía}

Álvarez, A. (2011). Medición y Evaluación en Comunicación (Segunda edición). Instituto de Investigación en Relaciones Públicas.

Álvarez, A. (2014). Manual del Estudiante. Quito. UTPL

Álvarez A. (2011). Medición y Evaluación en Comunicación. Málaga.

Bertalanffy Von, L. (1976). Teoría General de los Sistemas. Editorial Fondo de Cultura Económica. México.

Comunicación Asertiva. (2014). Obtenido de Comunicación Asertiva. Recuperado el día

14 de junio de 2014 de:

http://rero.jimdo.com/app/download/10613757657/comunicacion+2014+j.pdf?t=141704

$\underline{7574}$

Ministerio del ambiente. Plan estratégico MAE. Recuperado el día 20 de julio de 2014, de: www.ambiente.gob.ec/wp.../07/Plan-estratégico -MAE-2012.pdfEncachéSimilares

\section{Forma de citar este artículo en bibliografías}

VALDIVIEZO ABAD, Karen Cesibel y SOSA, María Teresa (2015): "Medición y evaluación en comunicación estratégica. Caso de estudio Ministerio del Ambiente del Ecuador", en Revista PANGEA № 6, Volumen № 2, páginas 01 a 12. Tenerife: Red Académica Iberoamericana de Comunicación. Recuperado el _ de de 2 de: http://www.revistapangea.org 